Check for updates

Cite this: RSC Adv., 2017, 7, 18601

Received 28th January 2017

Accepted 16th March 2017

DOI: $10.1039 / c 7 r a 01223 c$

rsc.li/rsc-advances

\section{Size-tunable uniform gold octahedra: fast synthesis, characterization, and plasmonic properties}

\begin{abstract}
Yonggang Lu, (D)*ab Haibin Zhang, (D)*ab Fan Wu, ${ }^{b}$ Hong Liu ${ }^{a}$ and Jingzhong Fang ${ }^{\text {ab }}$
A modified polyol process was developed to synthesize uniform and high-purity gold (Au) octahedra in a refluxing 1,5-pentanediol (PD) solution by introducing the cationic surfactant poly(diallyldimethylammonium) chloride (PDDA). Well-defined octahedral Au nanocrystals (NCs) with sizes ranging from 30 to $110 \mathrm{~nm}$ could be facilely and precisely prepared in high yield by controlling the experimental parameters. Our study indicates that the surfactant PDDA with the preferential adsorption on the $\{111\}$ planes of Au nuclei plays a crucial role for the controllable synthesis of uniform octahedral Au NCs. Moreover, the size-dependent optical properties of Au nanooctahedra were systematically investigated. The surface plasmon resonance (SPR) absorption peaks were red-shifted from 536 to $613 \mathrm{~nm}$ with the increasing sizes. It is promising that octahedral Au NCs with the size-dependent plasmonic properties are potential candidates for a broad range of applications related to optics, metamaterial, biomedicine, and catalysis.
\end{abstract}

\section{Introduction}

The optoelectronic and physicochemical properties of noble metal nanocrystals (NCs) are highly dependent on the morphology (shape and size). ${ }^{1-5}$ For instance, the position, quantity, and intensity of the SPR properties of noble metal NCs show a strong correlation with their morphologies. ${ }^{6,7}$ The development of selectively synthetic strategy for metal NCs with tailored shapes and sizes has received considerable attention in recent years due to providing an effective approach for precisely tuning the electronic, magnetic, optoelectronic, optical, and catalytic properties of the metal NCs. ${ }^{8-15} \mathrm{Au}$ NCs, one of the most important noble metal NCs, have been widely researched owing to their high chemical stability and intensive SPR properties. ${ }^{7,16}$ To date, a number of synthetic approaches have been developed to afford anisotropic Au NCs in the shapes of cubes, ${ }^{17}$ prisms,${ }^{18}$ plates,${ }^{19}$ decahedra, ${ }^{20}$ trisoctahedra, ${ }^{21}$ rods,${ }^{22,23}$ wires,${ }^{24}$ dendrites, ${ }^{25}$ etc. Among them, the polyol process, which has been frequently used to prepare anisotropic metal nanoparticles, is a versatile and convenient method for the controllable synthesis of Au NCs. ${ }^{2}$ In the polyol process, ethylene glycol normally serves as both solvent and reductant agent in the reaction mixture. It is well known that the formation of a particular shape of Au NCs requires the presence of a surfactant. Meanwhile, the surfactant can vary the order of free energies of different facets through their interactions with

${ }^{a}$ Lightweight Optics and Advanced Materials Center, Institute of Optics and Electronics, Chinese Academy of Sciences, Chengdu, 610209, P. R. China. E-mail: luygioe@163.com; zhb@ioe.ac.cn

${ }^{b}$ University of Chinese Academy of Sciences, Beijing, 100049, P. R. China the $\mathrm{Au}$ nuclei surface in the synthesis process, and thus significantly affect the relative growth rates of different crystal facets, lending to produce the final products with different morphologies.

In particular, the octahedral nanostructure is one of the ideal morphologies with eight equilateral triangle faces. Different from other polyhedral Au NCs, octahedral Au NCs have much lower surface free energy and relatively different plasmonic properties. So far, there are several reports on the synthesis of octahedral Au NCs by various approaches. ${ }^{26-32}$ To this end, Cho and co-workers synthesized octahedral Au NCs at $125{ }^{\circ} \mathrm{C}$ for $6-$ $48 \mathrm{~h}$ in the poly(ethylene glycol) 600 solution, where the surfactant poly(vinyl pyrrolidone) (PVP) was injected as stabilizer. $^{30}$ Song and co-workers demonstrated that the polyol process was developed to prepare octahedral Au nanoparticles by introducing $[\mathrm{Ag}]^{+}$ions in the presence of the surfactant PVP. ${ }^{31}$ In addition, Xia and co-workers reported the synthesis of $\mathrm{Au}$ nanooctahedra from 16 to $77 \mathrm{~nm}$ by seed-mediated growth in the presence of PVP at $80{ }^{\circ} \mathrm{C} .{ }^{32}$ Although the prior synthetic strategies were able to produce octahedral Au NCs, the relatively long reaction times, the introducing of foreign metal ions and the multistep process were necessary to selectively synthesize octahedral Au NCs. Therefore, it is highly desirable to develop a straightforward and effective synthetic strategy to fast generate high-purity Au nanooctahedra with tunable sizes for uncovering properties and achieving fascinating applications.

Herein, an one-pot modified polyol process could provide a feasible and effective route for the controllable synthesis of high-purity Au nanooctahedra by introducing the cationic surfactant PDDA in a refluxing 1,5-pentanediol (PD) solution rather than the traditional ethylene glycol. Owing to the 
excellent adsorption abilities on the particular crystal planes, the surfactant PDDA has been used as the capping reagent to afford anisotropic NCs in our previous reports. ${ }^{2,33,34}$ Meanwhile, the presence of PD as both solvent and reductant agent in the reaction mixture is very effective for the supply of higher temperature environment, due to its higher boiling temperature. Additionally, the fast synthetic process requires no seed preparation procedure and no further addition of foreign metal ions until the formation of octahedral Au NCs. High-purity Au nanooctahedra with tunable sizes were facilely and selectively synthesized only by tuning the initial experimental parameters (including the PDDA concentrations, the reaction temperatures, and the reaction time intervals). The size-dependent optical properties of $\mathrm{Au}$ nanooctahedra were systematically investigated in our study. Furthermore, the morphology and optical properties of the as-synthesized octahedral Au NCs displayed an excellent stability over a long period of time. In general, we would like to propose a new, straightforward and alternative modified polyol method for the controllable synthesis of uniform octahedral Au NCs with tunable sizes and interesting plasmonic properties.

\section{Materials and methods}

\subsection{Materials}

1,5-Pentanediol (PD, $\geq 97.0 \%$ ), gold(III) chloride trihydrate $\left(\mathrm{HAuCl}_{4} \cdot 3 \mathrm{H}_{2} \mathrm{O}, \geq 49.0 \%\right.$ Au basis), and poly(diallyldimethylammonium chloride) solution (PDDA, $M_{\mathrm{w}}=400000-$ $500000 \mathrm{D}, 20$ wt $\%$ in $\mathrm{H}_{2} \mathrm{O}, \geq 99.0 \%$ ) were all purchased from Sigma-Aldrich and used as received without further purification. The water used in our experiments was ultrapure deionized water (18.2 M $\Omega$ ).

\subsection{Synthesis of Au nanooctahedra}

For a representative synthesis of $\mathrm{Au}$ nanooctahedra, $0.16 \mathrm{~mL}$ of PDDA solution and $0.5 \mathrm{~mL}$ of $20 \mathrm{mM} \mathrm{HAuCl}_{4}$ solution in PD were sequentially introduced into $10 \mathrm{~mL}$ of $\mathrm{PD}$ solution in a glass vial under stirring at room temperature and ambient conditions. The final concentrations of $\left[\mathrm{AuCl}_{4}\right]^{-}$ions and PDDA in the initial gold precursor were about $1 \mathrm{mM}$ and $20 \mathrm{mM}$, respectively (the molar ratio of PDDA to $\left[\mathrm{AuCl}_{4}\right]^{-}$ions of 20). The resulting mixture was then capped tightly in the vial and refluxed at $220^{\circ} \mathrm{C}$ for 1 hour in an oil bath. During the reaction process, the color of solution gradually changed from yellow to colorless in the initial step. Afterward, a reddish color appeared, indicating the formation of $\mathrm{Au}$ NCs in the reaction mixture, and finally reddish brown ended at $1 \mathrm{~h}$. The final products were harvested by centrifugation (12 $000 \mathrm{rpm}, 30$ min) and washed with acetone once and with water twice to remove excess PDDA or PD. After the purification, the as-synthesized Au NCs were redispersed in deionized water for further characterizations and plasmonic property studies. In addition, further experiments by varying the experimental parameters (including the PDDA concentrations, the reaction temperature, and the reaction time intervals), were also conducted to determine the different influence factors on the controllable synthesis of typical octahedral Au NCs.

\subsection{Characterization}

Scanning electron microscopy (SEM) images of the Au NC samples were taken using a ZEISS ULTRA PLUS-43-12 operating at $15 \mathrm{kV}$. Transmission electron microscopy (TEM), highresolution TEM (HRTEM), and corresponding selected area electron diffraction (SAED) patterns were measured on a FEI Tecnai-G2 F30 at $300 \mathrm{kV}$. X-ray diffraction (XRD) data were obtained on a BRUKER D8 DISCOVER diffractometer equipped with the $\mathrm{Cu} \mathrm{K} \alpha$ radiation source $(\lambda=1.5418 \AA)$. UV-Vis-NIR absorption spectra were collected on a Lambda-1050 dual beam UV-Vis spectrophotometer by using the colloidal water suspension.

\section{Results and discussion}

\subsection{Structural characterization}

Fig. 1A and B show the typical low- and high-magnification SEM images of $\mathrm{Au}$ NCs synthesized in the representative synthesis. It can be seen that the Au NCs exhibited perfectly octahedral nanoconstructions in high yield (nearly 100\%) with sharp edges and corners as well as smooth surfaces. The average edge length of the regular $\mathrm{Au}$ nanooctahedra was $75 \mathrm{~nm}$ with a standard deviation of $2.5 \mathrm{~nm}$ (Fig. 1E). Fig. 1C displays the overview TEM image of the typical Au nanooctahedra. It indicates that the projection of Au nanoparticles was rhombus with uniform size. The HRTEM image and the corresponding SAED pattern (Fig. 1D), were performed on an individual Au octahedron (the square region in Fig. 1C), by focusing the electron beam perpendicular to the triangular facet of the nanoparticle. The diffraction spots with the six-fold rotational symmetry could be indexed to the $\{220\}$ reflections of face-centered cubic (fcc) $\mathrm{Au}$ nanoparticle. Moreover, the continuous fringe pattern with spacing of $0.237 \mathrm{~nm}$ on the surface of the $\mathrm{Au} \mathrm{NC}$ and the diffraction spots together demonstrated that each $\mathrm{Au}$ nanoparticle was a single crystal with $\{111\}$ lattice planes as the basal surfaces. Fig. $1 \mathrm{~F}$ presents the XRD pattern of such as-prepared $\mathrm{Au}$ NCs sample, which further reveals that the nanoparticles were crystalline face-centered cubic (fcc) Au with abundance of $\{111\}$ planes. Specifically, the obvious $\{111\}$ diffraction peak with exceptionally strong intensity relative to the $\{200\},\{220\}$, and $\{222\}$ diffraction peaks in the XRD spectrum, reflected the preferential orientation of the NCs along $\{111\}$ planes parallel to the supporting substrate. ${ }^{30}$ In general, these above results verified that the high-purity and uniform Au nanooctahedra completely covered by $\{111\}$ facets, could be easily obtained through the representatively modified polyol process in PD solution with the assistance of the cationic surfactant PDDA.

\subsection{Influence factor of the concentrations of PDDA}

In the representative synthesis strategy, $\mathrm{PD}, \mathrm{PDDA}$, and $\mathrm{HAuCl}_{4}$ served as solvent, polymeric capping agent, and salt precursor in the reaction mixture, respectively. Especially, it was anticipated that the surfactant PDDA was a crucial factor for the controllable and selective formation of high-purity $\mathrm{Au}$ nanooctahedra with uniform size. A series of experiments were performed by changing PDDA concentration in the initial 

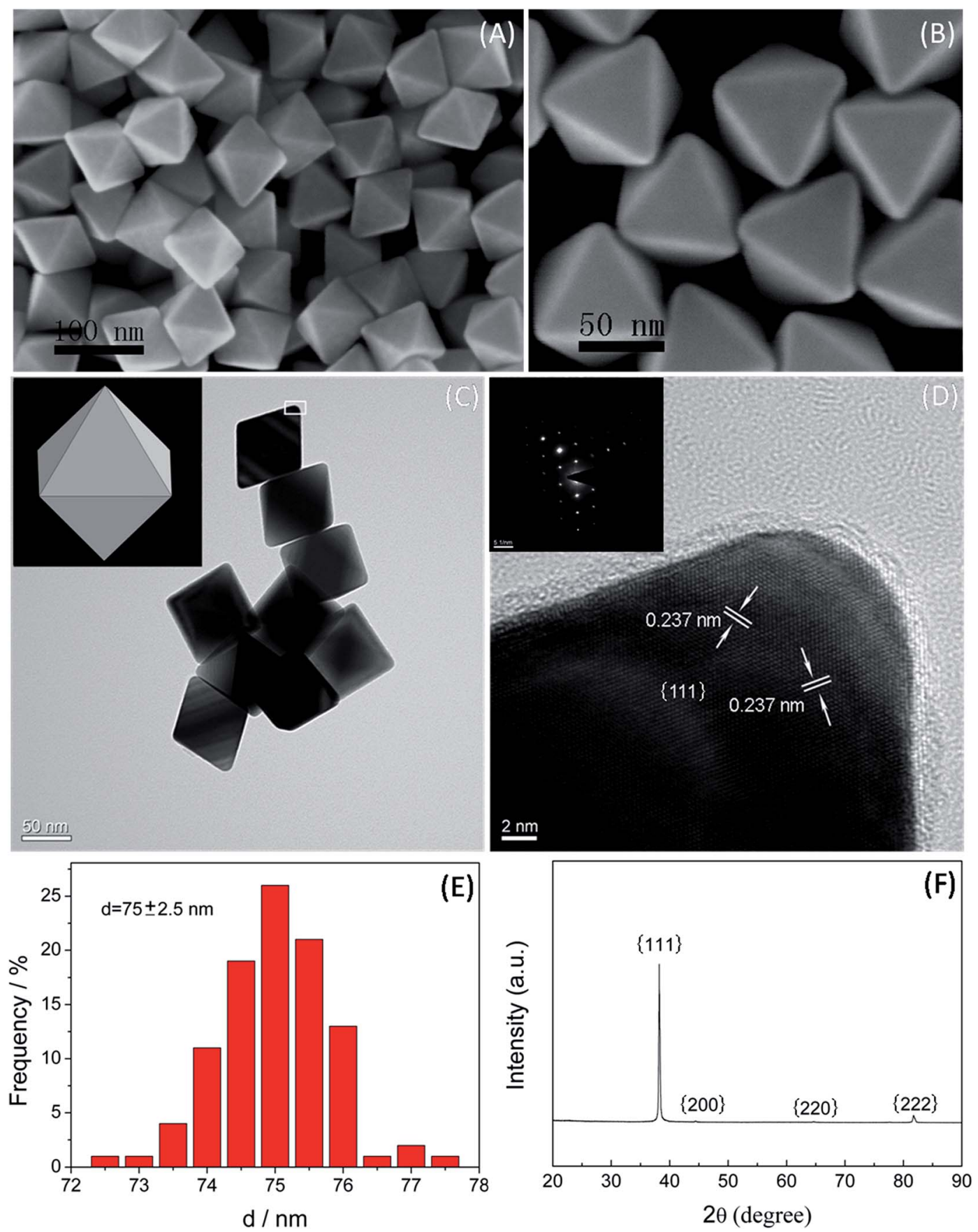

Fig. 1 (A) Low- and (B) high-magnification SEM images of the octahedral Au NCs prepared in the representative synthesis. (C) TEM image and the 3D model (inset) of octahedral Au NCs. (D) HRTEM image and the corresponding SAED pattern (inset) taken from an individual Au octahedron (the square region in $(C)$ ). The electron beam was perpendicular to the triangle face of the octahedral particle in (C). (E) Size distribution of the asprepared octahedral Au NCs. (F) X-ray diffraction pattern.

precursors and keeping other experimental parameters constant to reveal the importance of the surfactant PDDA. When the surfactant PDDA was absent in the reaction mixture, $\left[\mathrm{AuCl}_{4}\right]^{-}$ions were rapidly reduced to $\mathrm{Au}$ atoms, which were then aggregated to form irregular Au NCs (Fig. 2A). Interestingly, well-defined octahedral Au NCs was observed with the increase of the PDDA concentration, as shown in Fig. 2B-G. It can be seen that uniform Au nanooctahedra were produced, of which the mean edge length was $50 \mathrm{~nm}$ with a narrow size distribution (Fig. 2B), when the PDDA concentration was $5 \mathrm{mM}$ in the initial precursor (PDDA: $\mathrm{HAuCl}_{4}=5$ ). In addition, octahedral Au nanoparticles with the average edge lengths of 60 , $75,80,83$, and $105 \mathrm{~nm}$ were selectively prepared in high yield, while the PDDA concentrations were 10, 20, 30, 40, and $50 \mathrm{mM}$, and the corresponding molar ratios of PDDA to $\mathrm{HAuCl}_{4}$ were 10 , $20,30,40$, and 50 , respectively (Fig. 2C-G). Obviously, the sizes of octahedral Au NCs increased with the increase of the PDDA concentration in the initial precursors. However, when the 

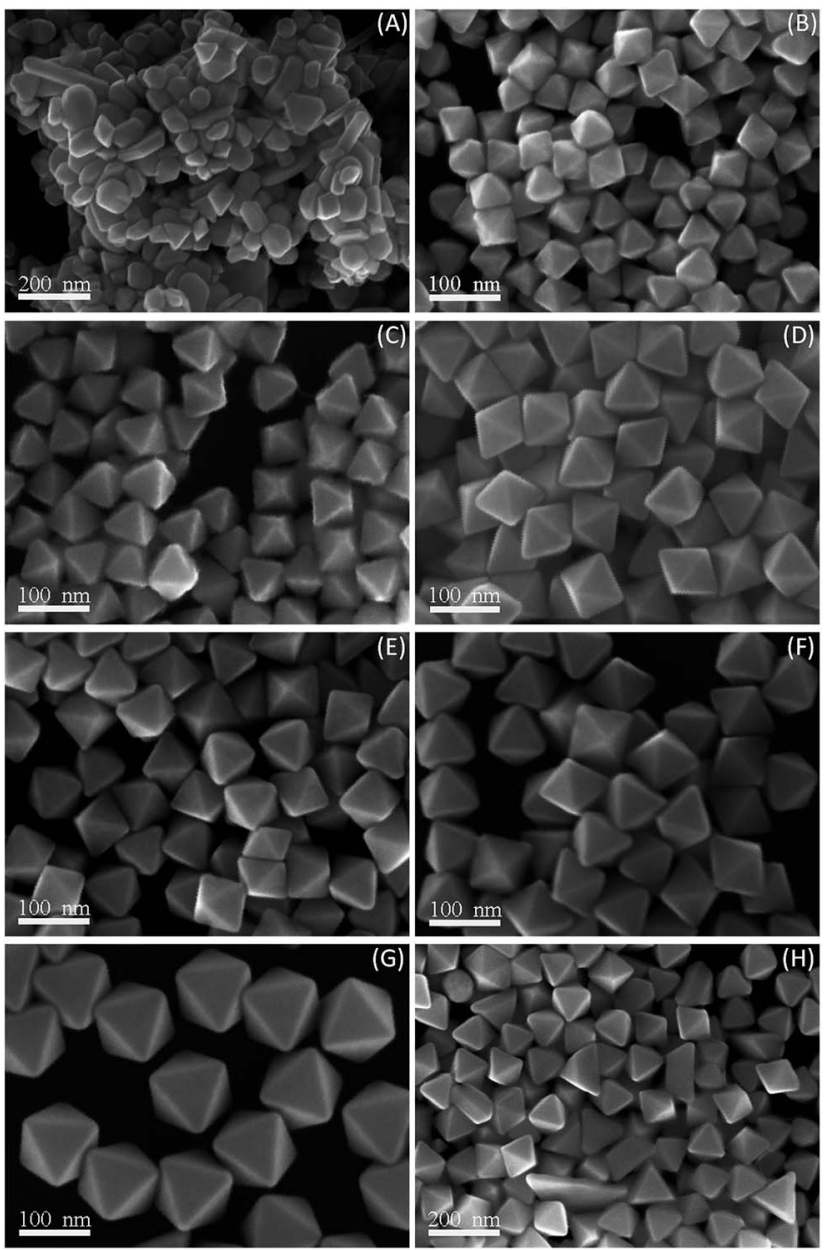

Fig. 2 SEM images of the Au NCs synthesized at $220^{\circ} \mathrm{C}$ for $1 \mathrm{~h}$ in PD solution containing $1 \mathrm{mM} \mathrm{HAuCl}_{4}$ and various concentrations of PDDA: (A) 0, (B) 5, (C) 10, (D) 20, (E) 30, (F) 40, (G) 50, and (H) $80 \mathrm{mM}$ in the reaction mixture, respectively.

PDDA concentration was further increased to $80 \mathrm{mM}$ (PDDA : $\mathrm{HAuCl}_{4}=80$ ), low-purity octahedral Au NCs (including several polyhedral Au NCs) were generated in the final products (Fig. 2H). This phenomenon was primarily attributed to high coverage of PDDA on several other planes of Au nuclei and thus generating the various crystal faces on the surface of Au NCs. It almost agreed with the previous reports on the shape evolution of metal nanoparticles by using PVP as the capping agent, where the nanoparticles usually evolved to other shapes at the high concentration of PVP. ${ }^{2}$ As described above, the cationic surfactant PDDA both as the stabilizer and as the strong shape controller was indispensable for the formation of high-purity $\mathrm{Au}$ nanooctahedra with uniform size. As a result, the modified polyol process in PD solution by introducing PDDA presented here was a straightforward and effective synthetic strategy for high-yield preparation of high-purity octahedral Au NCs with well-defined shape and tunable sizes.

The controllable synthesis of high-purity octahedral $\mathrm{Au}$ nanoparticles with tunable sizes by the various PDDA concentrations in the reaction mixture, allowed us to fully investigate the size-dependent optical properties of octahedral Au NCs.
Clearly, the characteristic SPR peaks were located at 553, 567, $582,590,600$, and $610 \mathrm{~nm}$, while $\mathrm{Au}$ nanoparticles were prepared by the PDDA concentrations of 5, 10, 15, 20, 25, and $50 \mathrm{mM}$, respectively (Fig. 3). The sharp absorption peaks could be primarily attributed to the uniform shape and size of the octahedral $\mathrm{Au}$ NCs. Moreover, As the PDDA concentration reached $80 \mathrm{mM}$, the broad SPR absorption peak was appeared at $616 \mathrm{~nm}$ mainly from low-purity Au nanooctahedra. Accordingly, as the size of the well-defined octahedral $\mathrm{Au}$ nanoparticles gradually increased, the SPR peak was red-shifted in the absorption spectra. This was complete the same as the previous reports on the SPR absorption peak of octahedral Au NCs, where the SPR peaks were red-shifted from 525 to $599 \mathrm{~nm}$ (the edge length from 16.2 to $76.8 \mathrm{~nm}) .^{32}$

\subsection{Influence factor of the reaction temperature}

In the modified polyol process, the crystal growth rate of octahedral $\mathrm{Au}$ nanostructures and the reducing power of $\mathrm{PD}$, is greatly influenced by the reaction temperature. PD possesses a higher boiling point $\left(242^{\circ} \mathrm{C}\right)$ than ethylene glycol $\left(197^{\circ} \mathrm{C}\right)$ and holds sufficient solubility for dissolving $\mathrm{Au}$ precursors and PDDA. Experiments at various reaction temperatures were thus performed without varying any other experimental parameters. At the low reaction temperature $\left(200{ }^{\circ} \mathrm{C}\right)$, octahedral $\mathrm{Au}$ nanoparticles with a broad size distribution, as well as polyhedra and irregular NCs, were produced (Fig. 4A). In this case, the insufficiently reducing power of $\mathrm{PD}$ at the relatively low reaction temperature, influenced the generation rate of source $\mathrm{Au}$ atoms, and finally went against the formation of uniform octahedral Au NCs. Otherwise, when the reaction temperature was increased ranging from 210 to $250{ }^{\circ} \mathrm{C}$, high-purity $\mathrm{Au}$ nanooctahedra were dominantly prepared. Meanwhile, the mean edge lengths of $\mathrm{Au}$ nanooctahedra were $110,75,65,52$, and $45 \mathrm{~nm}$ as the reaction temperatures were $210,220,230,240$, and $250{ }^{\circ} \mathrm{C}$, respectively (Fig. 4B-F). Apparently, the size of Au nanooctahedra decreased

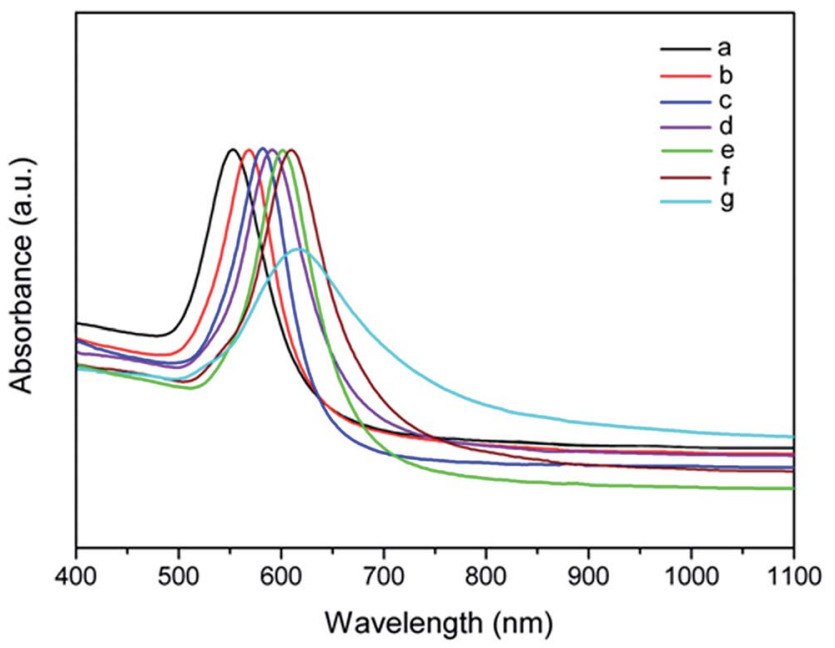

Fig. 3 UV-Vis-NIR absorption spectra of the Au NCs samples synthesized in PD solution containing $1 \mathrm{mM} \mathrm{HAuCl}_{4}$ and various concentrations of PDDA: (a) 5, (b) 10, (c) 20, (d) 30, (e) 40, (f) 50, and (g) $80 \mathrm{mM}$ in the reaction mixture, respectively. 

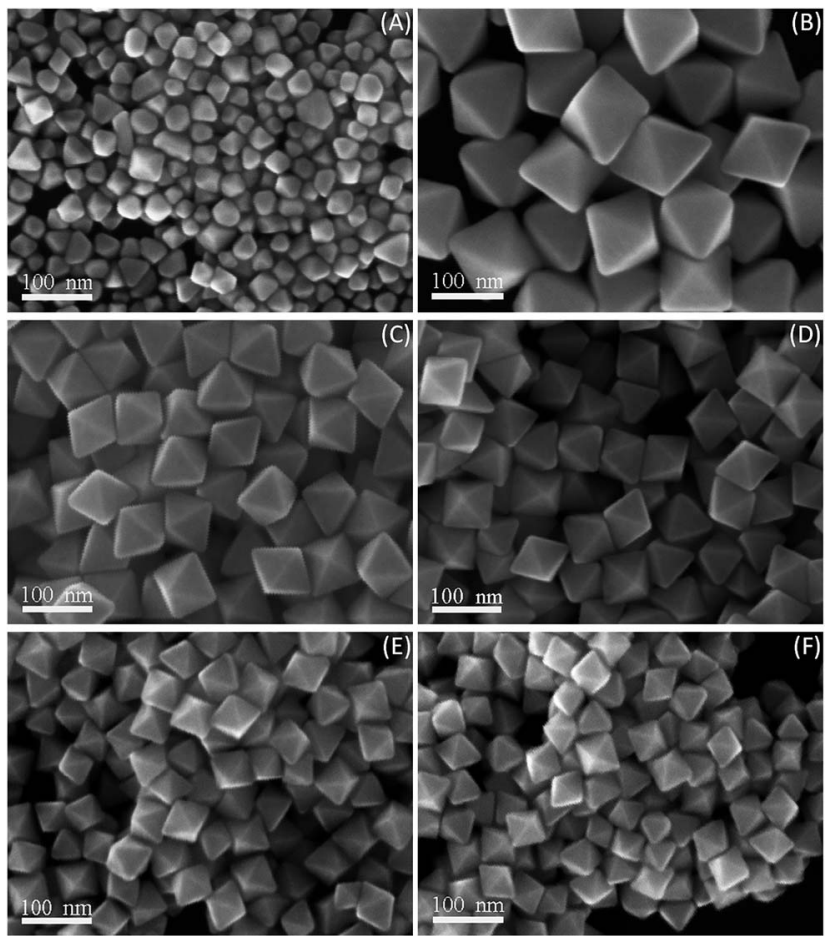

Fig. 4 SEM images of products generated at various reaction temperatures: (A) $T=200^{\circ} \mathrm{C}$, (B) $T=210^{\circ} \mathrm{C}$, (C) $T=220^{\circ} \mathrm{C}$, (D) $T=$ $230^{\circ} \mathrm{C}$, (E) $T=240{ }^{\circ} \mathrm{C}$, and (F) $T=250^{\circ} \mathrm{C}$, respectively.

with the increase of the reaction temperature. As such, the higher reaction temperature was propitious to enhance the generation rate of $\mathrm{Au}$ atoms, and thus decrease the size of the final octahedral $\mathrm{Au}$ NCs. As described above, the reaction temperature could kinetically manipulate the reduction rate of gold ions, and this in turn affected the initial gold nucleation process, which was one of determining factors for the morphology of the final products. Consequently, high-purity $\mathrm{Au}$ nanooctahedra can be generated in a wide reaction temperature region and the sizes of Au nanoparticles can be manipulated by precisely changing the reaction temperature.

Fig. 5 shows UV-Vis-NIR absorption spectra of the regular Au nanooctahedra generated at the various reaction temperatures. The sharp SPR peak was continuously blue-shifted by the increase of reaction temperature from 210 to $250{ }^{\circ} \mathrm{C}$. The characteristic SPR peaks were located at $613,582,573,557$, and $549 \mathrm{~nm}$, corresponding to the uniform $\mathrm{Au}$ nanooctahedra prepared at $210,220,230,240$, and $250{ }^{\circ} \mathrm{C}$, respectively. Nevertheless, when the reaction temperature was $200{ }^{\circ} \mathrm{C}$, another SPR peak with a relatively broad shape was appeared at $534 \mathrm{~nm}$, owing to low-purity Au nanooctahedra. The difference of the SPR absorption properties of Au octahedra generated at the various reaction temperatures was consistent with that by varying PDDA concentrations in the initial precursor.

\subsection{Formation mechanism}

The formation process is always an intensive topic of the research on anisotropic Au NCs. During the octahedral

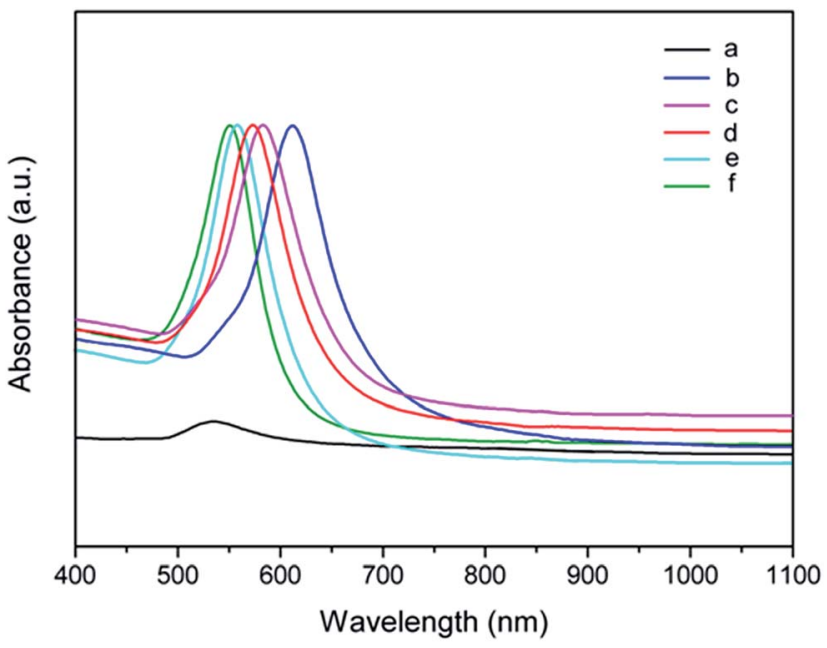

Fig. 5 UV-Vis-NIR absorption spectra of the Au NCs samples generated at various reaction temperatures: (a) $T=200^{\circ} \mathrm{C}$, (b) $T=210^{\circ} \mathrm{C}$, (c) $T=220^{\circ} \mathrm{C}$, (d) $T=230{ }^{\circ} \mathrm{C}$, (e) $T=240{ }^{\circ} \mathrm{C}$, and (f) $T=250{ }^{\circ} \mathrm{C}$, respectively.

synthetic process, the reactions were quenched at various time intervals to investigate the mechanism related to the evolution of the shapes and sizes of octahedral Au NCs. The color of the reaction mixture changed from yellow to colorless, and to reddish in the initial step (about $3 \mathrm{~min}$ ). The SEM observation displays that octahedral Au nanoparticles with a little broad size distribution (edge lengths ranging from 30 to $40 \mathrm{~nm}$ ) were exclusively produced (Fig. 6A). This could reveal that the $\mathrm{Au}(\mathrm{III})$
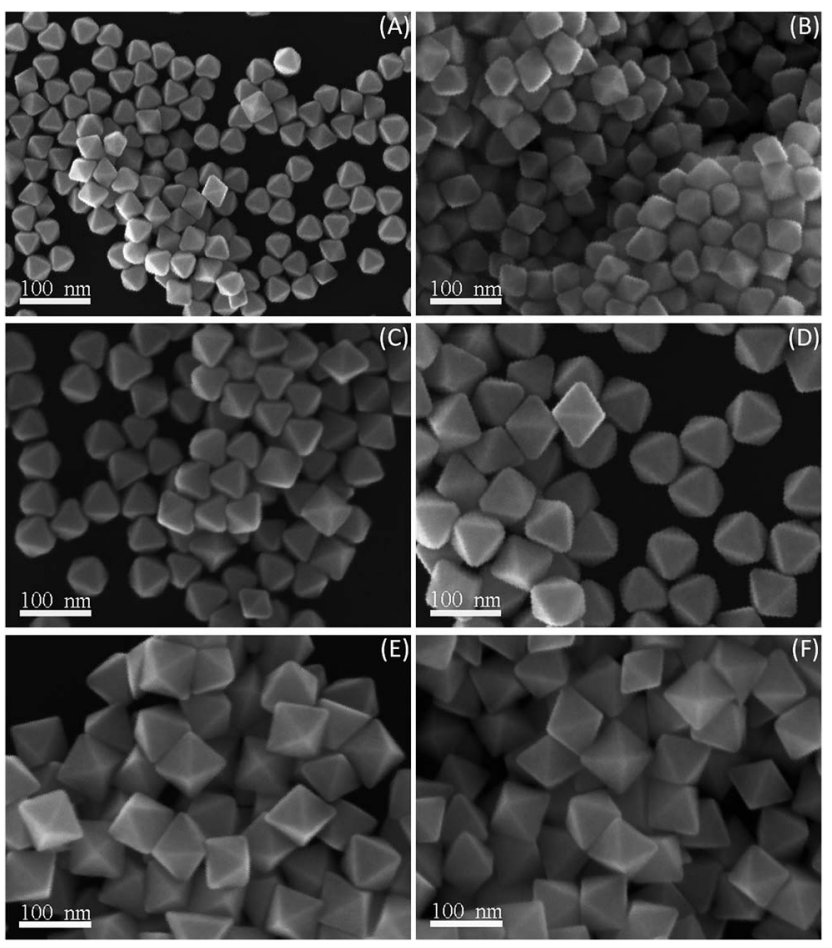

Fig. 6 SEM images of octahedral Au NCs obtained at different reaction time intervals: (A) $3 \mathrm{~min},(B) 10 \mathrm{~min},(C) 20 \mathrm{~min}$, (D) $30 \mathrm{~min}$, (E) $2 \mathrm{~h}$, and (F) $5 \mathrm{~h}$, respectively. 


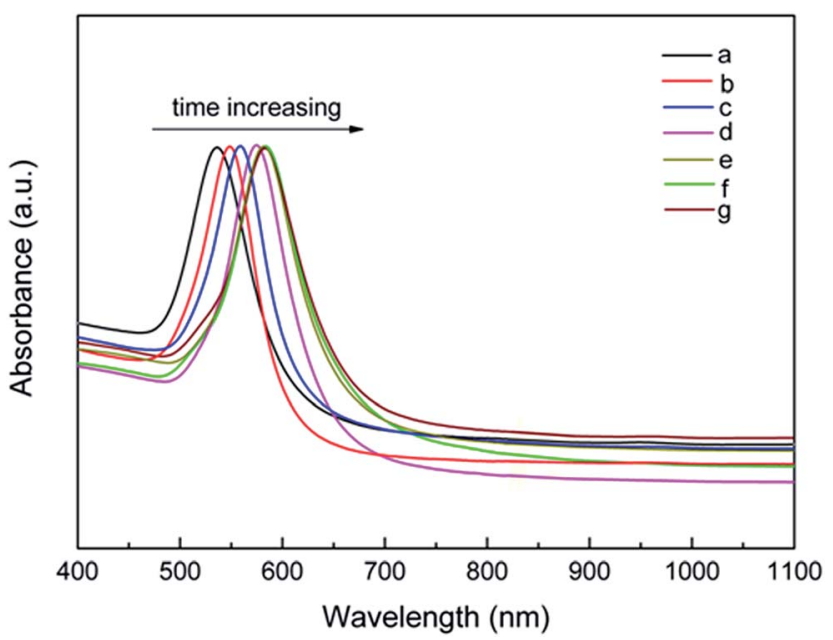

Fig. 7 UV-Vis-NIR absorption spectra of octahedral Au NCs obtained at different reaction time intervals: (a) 3 min, (b) 10 min, (c) 20 min, (d) $30 \mathrm{~min},(\mathrm{e}) 1 \mathrm{~h}$, (f) $2 \mathrm{~h}$, and (g) $5 \mathrm{~h}$, respectively.

ions in the precursor were directly converted into zero-valent $\mathrm{Au}(0)$ atoms in the PD solution under higher reaction temperature rather than a multistep process from $\mathrm{Au}(\mathrm{III})$ to $\mathrm{Au}(\mathrm{I})$ and eventually to $\mathrm{Au}(0)$ in the EG solution. As the reaction went on, the mean edge length of Au nanooctahedra gradually increased. The average edge lengths of nanooctahedra harvested at 10, 20, and $30 \mathrm{~min}$ were 43,52 , and $66 \mathrm{~nm}$ with a narrow size distribution, respectively (Fig. 6B-D). The corresponding color evolution ranging from reddish to reddish brown was clearly observed. Subsequently, when the reaction time interval reached $2 \mathrm{~h}$ and $5 \mathrm{~h}$, the color of the final reaction solution kept reddish brown. The SEM examinations present that the uniform $\mathrm{Au}$ nanooctahedra obtained at 2 and $5 \mathrm{~h}$ had nearly the same edge length of $75 \mathrm{~nm}$ (Fig. 6E and F). In this case, Ostwald ripening that smaller seeds dissolved and larger particles grew to uniform sizes, might occur concomitantly with the process of $\mathrm{Au}$ nanoparticles growth to afford the final products with uniform size. Accordingly, Au nanooctahedra with tunable sizes $(30-75 \mathrm{~nm})$ could be also selectively harvested by controlling the reaction time. These results indicated that, besides the PDDA concentration and the reaction temperature, the size of $\mathrm{Au}$ nanooctahedra can be rationally manipulated over a broad range by varying the reaction time intervals.

Fig. 7 displays the typical UV-Vis-NIR absorption spectra of uniform $\mathrm{Au}$ nanooctahedra prepared by the various reaction time intervals. As the reaction time increased from $3 \mathrm{~min}$ to $5 \mathrm{~h}$, the corresponding edge lengths of uniform Au nanooctahedra gradually increased from 30 to $75 \mathrm{~nm}$, and their SPR absorption peaks were red-shifted from 536 to $582 \mathrm{~nm}$. Three almost identical SPR absorption peak positions of Au nanooctahedra at 1,2 , and $5 \mathrm{~h}$, could further reveal that the growth process of octahedral Au NCs lasted approximately $1 \mathrm{~h}$. Similarly, the SPR absorption peak position of the uniform Au nanooctahedra was red-shifted with the increasing edge length, which was consistent with the previous result from the PDDA concentration and the reaction temperature. Additionally, the sharp absorption peaks further confirmed that the obtained Au nanooctahedra were uniform in the morphologies.

It is widely known that the ratio $(R)$ of the growth rate along the $\langle 100\rangle$ to the $\langle 111\rangle$ direction mainly determines the finally geometrical morphology of a cubical crystal. ${ }^{35}$ An ideally octahedral Au NC $(R=1.73)$ bounded by the highly stable $\{111\}$ facets is controllably generated, as a result of much faster growth rate of the $\langle 100\rangle$ direction than that of the $\langle 111\rangle$ direction. In general, with the assistance of selective adsorption of a surfactant, the crystal growth rates along the various directions can be precisely varying. Consequently, the introduction of a surfactant with the selective adsorption on a certain crystal plane is widely used to guide the synthesis of anisotropic metal nanoparticles in a solution phase. The surfactant PDDA is used as the capping agent to make anisotropic metal NCs stable by the strongly electrostatic binding to various metal nanostructure surfaces. In our experiment system, from the HRTEM image and the SAED pattern of the Au NCs (Fig. 1D), we could suppose that the surfactant PDDA was introduced to selectively adsorb on the $\{111\}$ crystal plane of Au NCs. Afterwards, the $\{100\}$ planes gradually disappeared while the $\{111\}$ planes became more dominant, and eventually generated well-defined $\mathrm{Au}$ nanooctahedra. Accordingly, the surfactant PDDA played a key role in the formation of octahedral Au nanoparticles. In
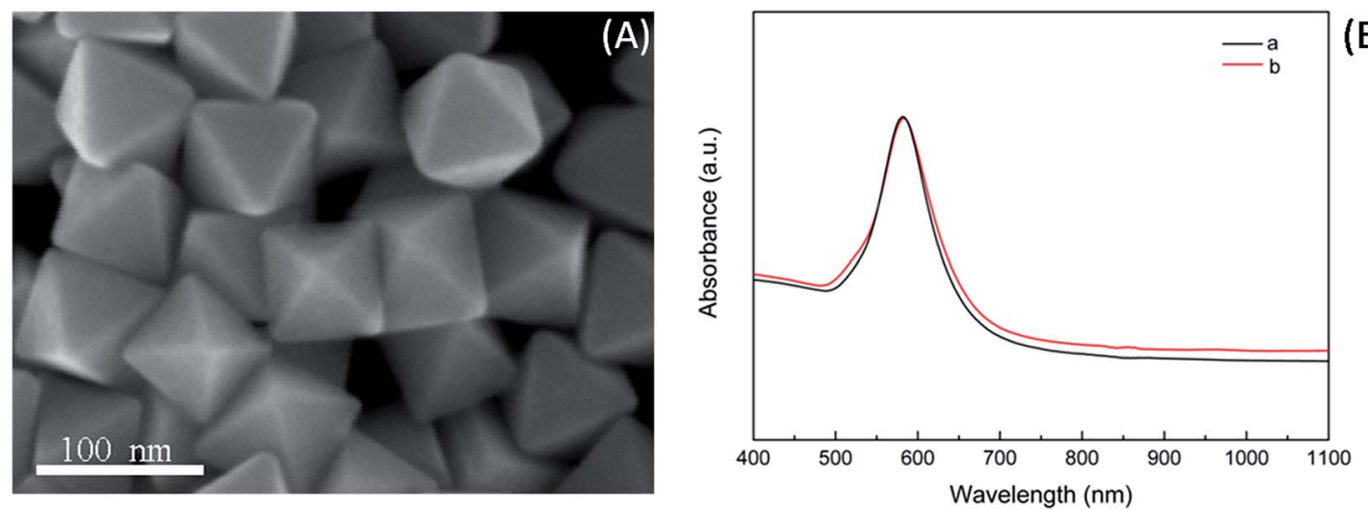

(B)

Fig. 8 (A) SEM image of the octahedral Au NCs prepared in the representative synthesis after being stored in water for three months. (B) UV-VisNIR plasmonic absorption spectra of octahedral Au NCs samples: (a) as-synthesized, and (b) after being stored for three months. 
addition, the surfactant PDDA is a cationic polymer and thus an initial strong electrostatic interaction between $[\mathrm{PDDA}]^{+}$ions and $\left[\mathrm{AuCl}_{4}\right]^{-}$ions results in the formation of stable ion pairs in the reaction mixture. The reduction rate of $\left[\mathrm{AuCl}_{4}\right]^{-}$ions in the modified polyol synthesis of Au nanooctahedra was decreased by the stable ion pairs. Previous results indicated that the slow reaction was propitious to the anisotropic growth of metal nanoparticles in the presence of shape controlled polymers. ${ }^{36} \mathrm{In}$ the slow process, the growth of Au nuclei could be kinetically controlled by the selective adsorption of the surfactant PDDA in the reaction mixture. On the basis of our analysis and results, we can concluded that the capping PDDA selectively adsorbed on the $\{111\}$ facets of Au nuclei, and then facilitated the growth of $\mathrm{Au}$ NCs along the $\langle 100\rangle$ direction, which leaded to the formation of well-defined octahedral Au NCs of uniform sizes. Moreover, the change in the morphology and plasmonic properties of $\mathrm{Au}$ nanooctahedra synthesized in the representative synthesis, did not occur after being stored for three months (Fig. 8). Therefore, we could conclude that Au nanooctahedra synthesized by our method were excellently stable owing to the electrostatic repulsion offered by the cationic surfactant PDDA.

Furthermore, the relatively high reaction temperatures (above $200{ }^{\circ} \mathrm{C}$ ) favored the formation of more uniform singlecrystal products, nevertheless the formation of less stable twinned particles such as decahedra also surrounded by $\{111\}$ planes, was suppressed. ${ }^{2}$ It was suggested that the reductive process at relatively high temperature provided appropriate conditions for controllable formation of octahedral $\mathrm{Au}$ NCs. Meanwhile, oxidative etching by the $\mathrm{O}_{2} / \mathrm{Cl}^{-}$pair occurred in the reaction process, which was also propitious to selectively remove the twinned nanoparticles to gain single-crystal $\mathrm{Au}$ nanoparticles in high-purity. ${ }^{37}$

\section{Conclusions}

In summary, high-purity octahedral Au NCs were fast synthesized by the modified polyol process in a refluxing PD solution. Au nanooctahedra with uniform sizes ranging from 30 to $110 \mathrm{~nm}$ were able to be selectively and reproducibly synthesized in high yield by using the particular surfactant PDDA both as the stabilizer and as the shape controller. Structural characterizations by SEM, XRD, TEM, and HRTEM verified that Au nanooctahedra with $\{111\}$ lattice planes as the basal surfaces, were generated with sharp corners and smooth surfaces. The formation of welldefined Au nanooctahedra was primarily attributed to the preferential adsorption of PDDA on the $\{111\}$ planes of Au nuclei that inhibited the growth along the $\langle 111\rangle$ direction. Well-defined $\mathrm{Au}$ nanooctahedra with a series of tunable sizes were selectively synthesized only by tuning the initial experimental parameters (including the PDDA concentrations, the reaction temperatures, and the reaction time intervals). The size-dependent plasmonic properties of Au nanooctahedra were systematically investigated. The SPR absorption peak of the Au nanocrystals was red-shifted with the increase of edge length. Au nanooctahedra with the interesting plasmonic properties are potential candidates for wide applications related to optics, metamaterial, biomedicine, and catalysis in the future.

\section{Acknowledgements}

This work was supported from the Lightweight Optics and Advanced Materials Center (LOMC). We thank Prof. J. J. Zhou and Prof. Y. Y. Zhang for their additional insights.

\section{Notes and references}

1 Y. Sun and Y. Xia, Science, 2002, 298, 2176-2179.

2 Y. Xia, Y. Xiong, B. Lim and S. E. Skrabalak, Angew. Chem., Int. Ed., 2009, 48, 60-103.

3 A. R. Tao, S. Habas and P. Yang, Small, 2008, 4, 310-325.

4 P. Zijlstra, J. W. M. Chon and M. Gu, Nature, 2009, 459, 410413.

5 H. Song, F. Kim, S. Connor, G. Somorjai and P. Yang, J. Phys. Chem. B, 2005, 109, 188-193.

6 A. Tao, P. Sinsermsuksakul and P. Yang, Angew. Chem., Int. Ed., 2006, 45, 4597-4601.

7 X. Ye, L. Jin, H. Caglayan, J. Chen, G. Xing, C. Zheng, V. Doan-Nguyen, Y. Kang, N. Engheta, C. R. Kagan and C. B. Murray, ACS Nano, 2012, 6, 2804-2817.

8 F. Tao, Angew. Chem., Int. Ed., 2016, 55, 15212-15214.

9 R. Rajendra, P. K. Gangadharan, S. Tripathi, S. Kurungot and N. Ballav, Nanoscale, 2016, 8, 19224-19228.

10 G. Bae and C. M. Aikens, J. Phys. Chem. C, 2015, 119, 2312723137.

11 B. Fleury, R. Cortes-Huerto, O. Tache, F. Testard, N. Menguy and O. Spalla, Nano Lett., 2015, 15, 6088-6094.

12 P. Yan, R. Wang, N. Zhao, H. Zhao, D. Chen and F. Xu, Nanoscale, 2015, 7, 5281-5291.

13 H. You, X. Liu, H. Liu and J. Fang, CrystEngComm, 2016, 18, 3934-3941.

14 N. Tian, Z. Zhou, S. Sun, Y. Ding and Z. Wang, Science, 2007, 316, 732-735.

15 L. Polavarapu, S. Mourdikoudis, I. Pastoriza-Santos and J. Perez-Juste, CrystEngComm, 2015, 17, 3727-3762.

16 M. Grzelczak, J. Perez-Juste, P. Mulvaney and L. M. LizMarzan, Chem. Soc. Rev., 2008, 37, 1783-1791.

17 H. Wu, C. Kuo and M. H. Huang, Langmuir, 2010, 26, 1230712313.

18 J. Millstone, S. Park, K. Shuford, L. Qin, G. Schatz and C. Mirkin, J. Am. Chem. Soc., 2005, 127, 5312-5313.

19 M. B. E. Griffiths, S. E. Koponen, D. J. Mandia, J. F. McLeod, J. P. Coyle, J. J. Sims, J. B. Giorgi, E. R. Sirianni, G. P. A. Yap and S. T. Barry, Chem. Mater., 2015, 27, 6116-6124.

20 A. Sanchez-Iglesias, I. Pastoriza-Santos, J. Perez-Juste, B. Rodriguez-Gonzalez, F. J. G. de Abajo and L. M. LizMarzan, Adv. Mater., 2006, 18, 2529-2534.

21 A. Maity, A. Maiti, B. Satpati, A. Patsha, S. Dhara and T. K. Chini, J. Phys. Chem. C, 2016, 120, 27003-27012.

22 X. Kou, S. Zhang, Z. Yang, C. Tsung, G. D. Stucky, L. Sun, J. Wang and C. Yan, J. Am. Chem. Soc., 2007, 129, 6402-6404.

23 Y. Xiang, X. Wu, D. Liu, L. Feng, K. Zhang, W. Chu, W. Zhou and S. Xie, J. Phys. Chem. C, 2008, 112, 3203-3208.

24 Y. Huang, A. R. Ferhan, S. Cho, H. Lee and D. Kim, ACS Appl. Mater. Interfaces, 2015, 7, 17582-17586. 
25 H. Zhang, Y. Lu, H. Liu and J. Fang, RSC Adv., 2014, 4, 3675736764.

26 L. Wei, B. Lu, M. Sun, N. Tian, Z. Zhou, B. Xu, X. Zhao and S. Sun, Nano Res., 2016, 9, 3547-3557.

27 S. Gomez-Grana, C. Fernandez-Lopez, L. Polavarapu, J. Salmon, J. Leng, I. Pastoriza-santos and J. Perez-Juste, Chem. Mater., 2015, 27, 8310-8317.

28 C. Li, F. Fan, B. Yin, L. Chen, T. Ganguly and Z. Tian, Nano Res., 2013, 6, 29-37.

29 M. L. Personick, M. R. Langille, J. Zhang and C. A. Mirkin, Nano Lett., 2011, 11, 3394-3398.

30 C. Li, K. L. Shuford, Q. H. Park, W. Cai, Y. Li, E. J. Lee and S. O. Cho, Angew. Chem., Int. Ed., 2007, 46, 3264-3268.
31 D. Seo, C. I. Yoo, J. C. Park, S. M. Park, S. Ryu and H. Song, Angew. Chem., Int. Ed., 2008, 47, 763-767.

32 D. Y. Kim, W. Li, Y. Ma, T. Yu, Z. Li, O. O. Park and Y. Xia, Chem.-Eur. J., 2011, 17, 4759-4764.

33 H. Zhang, Y. Lu, H. Liu and J. Fang, Nanoscale, 2015, 7, 11591-11601.

34 H. Zhang, Y. Lu, C. Guan, N. Song, Y. Zhang, H. Liu and J. Fang, J. Mater. Chem. C, 2017, 5, 645-653.

35 Z. Wang, J. Phys. Chem. B, 2000, 104, 1153-1175.

36 M. Tsuji, M. Hashimoto, Y. Nishizawa, M. Kubokawa and T. Tsuji, Chem.-Eur. J., 2005, 11, 440-452.

37 B. Lim, Y. Xiong and Y. Xia, Angew. Chem., Int. Ed., 2007, 46, 9279-9282. 